\title{
On the Ehrenfest Paradox in the Bohr Atomic Model toward the Quantization of Gravitation
}

\author{
Fima Ardianto Putra
}

\begin{abstract}
Ehrenfest Paradox has been studied in the Bohr Atomic Model as the theoretical procedure such a way that we can express the coordinate curvature i.e. gravitational aspect in the electromagnetic coupling constant. The strength of the curvature depends on the principal quantum number which shows that the value of curvature is quantized. For $n=1$, the value is $0,00152 \times 10^{20} \mathrm{~m}^{-2}$. The curvature value in the Bohr atomic model can be a standard to measure how strong the curvature of all system are, by comparing them with this value. We also get the understanding that the change of the curvature $\delta R$ will manifest the curvature propagation in the form of quantum energy, i.e. $\delta \varepsilon=\hbar \kappa \delta R$. This theory can be considered to enlarge the unification theory between quantum and gravitation. Another consequence of this theory is the quantization of Equivalence Principle.
\end{abstract}

Keywords: curvature; coupling constant; quantization

\section{Introduction}

Bohr atomic model was built based on the Niels Bohr's idea in 1913 which had shown quantization of energy, anguler momentum, and electron orbit. This model can explain discrete spectrum of Hidrogen atom that shows energi quantization and it is viewed as the consistent atomic model after the Rutherford one. Nevertheless this atomic model cannot explain the fine structure appearance in every energy levels. Several times after, Bohr-Sommerfeld atomic model was built and successfull in explaining the fine structure. This model was built by considering that the electron mass will variate with respect to it's velocity as the consequence of relativitistic effect. This effect is understood as eliptic orbit with Thomas precision phenomenon of the electron when it is moving around the proton, such a way that it gives energy correction to the Bohr's model as follow [1]

$$
E_{n}=\frac{-m_{0} Z^{2} e^{4}}{\left(4 \pi \varepsilon_{0}\right)^{2} 2 n^{2} \hbar^{2}}\left[1+\frac{\alpha^{4} Z^{2}}{n}\left(\frac{1}{n_{\theta}}-\frac{3}{4 n}\right)\right]
$$

The difference of energy value in the Bohr-Sommerfeld model with respect to the Bohr one is stated by second term of Eq.(1), with $\alpha=\frac{1}{137}$ is the fine structure constant [1]. It shows how much ratio of electron's

Correspondence: ardiantoputraf2@gmail.com

Department of Physics, Universitas Jenderal Soedirman, JI. Dr. Soeparno

61, Kampus UNSOED Karangwangkal, 53123, Purwokerto, Indonesia

Full list of author information is available at the end of the article

${ }^{*}$ Equal contributor speed with respect to the light's speed. That term shows relativistic correction of energy in the Bohr atomic model.

On another hand, the state of Hydrogen atom was described by Dirac equation. In this equation, electron is not viewed that it has eliptic orbit like in the Bohr-Sommerfeld model, but it has intrinsic spin which is coupled with it's orbital momentum. This phenomenon is believed can explain the fine structure existence in the Hydrogen discrete spectrum exactly, with the energy value as follow [1]

$$
E_{n}=\frac{-m_{0} Z^{2} e^{4}}{\left(4 \pi \varepsilon_{0}\right)^{2} 2 n^{2} \hbar^{2}}\left[1+\frac{\alpha^{4} Z^{2}}{j}\left(\frac{1}{j_{\theta}}-\frac{3}{4 n}\right)\right]
$$

The second term in this equation has the same value with the second term in Eq.(2). Constants $\alpha$ is also called as coupling constants. Either Bohr-Sommerfeld model or Dirac one consistently can explain the fine structure existence as the relativistic effect of Hydrogen atom.

Although based on the experiment, the fine structure had clearly shown relativistic correction, but in this study theoretically we can built atomic model with considering Ehrenfest paradox [2]. This paradox relates to the space curvature according to the Einstein's point of view. So we can built understanding about how to see the Bohr atomic model in the curved space. Space curvature will be viewed as relativistic effect which replaces the fine structure existence in the Hydrogen spectrum. From here, there is an indication 
that space curvature will be quantized such a way that guides us to the concept of quantization of gravitation. This study will give the picture about the procedure of curvature quantization by including Ehrenfest paradox in the Bohr atomic model. Hence we can obtain relation between the fine structure and space curvature which represents gravitation. Then we will know how strong the gravitation in the electromagnetic's coupling constant representation is?. It perhaps open the way for seeing gravitational strength with respect to other kinds of interaction in the curvature representation.

\section{Linier Momentum in the Curved Space}

This study does not consider about how the dynamical aspect, such as how about the force between proton and electron in the curve coordinate, how about the stress-strain of the virtual disk, etc. It just exhibits the kinematical aspect, i.e. Lorentz Fitzgerald contraction because of rotational movement and using Einstein point of view to explain the paradox.

Suppose that electron moves around the nucleus with speed $v_{n}=\omega_{n} r_{n}$ close to $c$. Linier momentum for this speed is $p_{n}=\frac{m_{0} \omega_{n} r_{n}}{\sqrt{1-\frac{\left(\omega_{n} r_{n}\right)^{2}}{c^{2}}}}=\frac{h}{\lambda_{n}}$. Now we make equivalence between particle picture and wave picture for electron that equation, by defines $\lambda_{n}$ as follow:

$$
p_{n}=\frac{m_{0} \omega_{n} r_{n}}{\sqrt{1-\frac{\left(\omega_{n} r_{n}\right)^{2}}{c^{2}}}}=\frac{h}{\sqrt{1-\frac{\left(\omega_{n} r_{n}\right)^{2}}{c^{2}}} \lambda_{n}^{o}}
$$

When $v_{n}=\omega_{n} r_{n}$ close to $c$, the value of $\sqrt{1-\frac{\left(\omega_{n} r_{n}\right)^{2}}{c^{2}}} \approx 0$ and the value of $\lambda_{n}^{o} \approx \lambda_{0}$. Here, $\lambda_{0}$ is the Compton wave length of the electron. Through this manner, we will be easier to check the equivalence between particle and wave picture. It will be used to the next step for make de Broglie wave analysis of electron along the orbit to be easy.

Previously, in the non-relativistic Bohr atomic model, de Broglie wave length $\lambda_{n}$ is connected with circumference $\odot_{n}=2 \pi r_{n}$ as the length of the orbit is taken by the electron when it is moving around the proton. It is stated according to the relation $n \lambda_{n}=$ $2 \pi r_{n}[3]$.

It means that de Broglie wave length of electron and the circumference have a fixed value for a such $n$ [3]. For non-relativistic case, we can write $n \lambda_{n}=2 \pi r_{n}$ based on the Eq. (3), hence

$$
n \lambda_{n}^{o}=2 \pi r_{n}
$$

The writting like this shows that the relativistic effect is not considered yet by no seeing Lorentz factor affection.
We will show proton and electron condition by making extrapolation of the disk as their own reference frame. The $\odot_{e}$ is the circumference of electron orbit which bounds electron's disk frame while $\odot_{p}$ is the circumference which bounds the proton's disk frame. If electron speed is $v_{n} \approx c$, observer who stays with the proton looks the circumference of proton's disk is $2 \pi r_{n}$. Nevertheless at the same time, he looks the circumference of electron's disk $\odot_{e}$ contracts. The length of this circumference is shorter than $2 \pi r_{n}$ according to Lorent-Fitzgerald contraction. It is what we call as Ehrenfest Paradox which shows that $[2,4] \odot_{e} \neq 2 \pi$. Hence, the electron now is in the Non-Euclidean space.

The length of circumference $\odot_{p}$ with respect to the circumference $\odot_{e}$ must be as great as the lorentz factor. It means that the lorentz factor is the factor which will make $2 \pi$ to be smaller: $\odot_{e}=$ $\left(\sqrt{1-\frac{\left(\omega_{n} r_{n}\right)^{2}}{c^{2}}} 2 \pi\right) r_{n}$. Because $r_{n}$ does not contracts, the $r_{n}$ value for relativistic atomic Bohr is equal to it's non-relativistic, i.e. $a_{0} n^{2}$. Nevertheless, radius with that value will be viewed curved as the consequence of the curve space where the atomic model is in.

If we return to Eq. (4), so the equation does not yet perfectly show relativistic condition because the Lorentz factor is not yet entered, whereas the Lorentz factor becomes significant in the relativistic limit. If the Lorentz factor takes in to account in Eq. (4), so of course that de Broglie wave length will be shorter like in Eq.(4). This phenomenon must be balanced by circumference contraction in the right hand side. According to Ehrenfest paradox, the $2 \pi r_{n}$ value will be $\left(\sqrt{1-\frac{\left(\omega_{n} r_{n}\right)^{2}}{c^{2}}} 2 \pi\right) r_{n}$. It means that now we can understand that $\lambda_{n}^{\circ}$ as the contraction of $\lambda_{n}^{\circ}$ following the contraction of $2 \pi r_{n}$. Hence we get the relation

$$
\begin{aligned}
n\left(\sqrt{1-\frac{\left(\omega_{n} r_{n}\right)^{2}}{c^{2}}} \lambda_{n}^{\circ}\right) & =\left(\sqrt{1-\frac{\left(\omega_{n} r_{n}\right)^{2}}{c^{2}}} 2 \pi\right) r_{n} \\
n \lambda_{n} & =\phi_{n} r_{n}
\end{aligned}
$$

We see that the coordinate curvature will be quantized because curvature is the function of $n$. It is not found in both Bohr-Sommerfeld and Dirac model. When atomic exitation is going on, electron will changes from a such curvature to other curvatures.

\section{Bohr Atomic Curvature}

Curvature measurement will be started with introduce the modelling of two circumference as follow The smaller disk shows the circumference of the Bohr radius $r_{a}$ which is rotating with a such speed $v_{a}$. 


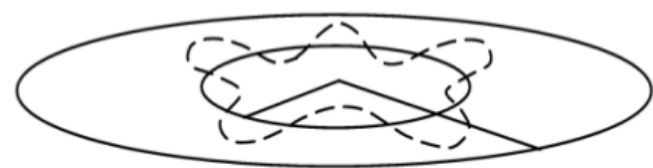

Figure 1: The small disk (Bohr circumference) with radius $r_{a}$ and the big disk with radius $r_{b}$ rotate with the same anguler velocity. De Broglie wave (e.g. for $\mathrm{n}=5$ ) is around the small disk.

Further we can make extrapolation of circumference with the radius $r_{b}$ which is rotating with the speed $v_{b}$, such a way that both of them obey the relation $\omega_{a}=\omega_{b}$ and so $\frac{v_{a}}{r_{a}}=\frac{v_{b}}{r_{b}}[5,6]$.

Suppose that for a such $r_{b}$ value, the value of $v_{b}=c$, so the circumference of biger disk will contract up to zero. It means that the area of the biger disk will be equal to the such of sphere with radius $R$. While the circumference of smaller disk will just contract up to a such value which is not zero, because $v_{a} \ll 0$. Based

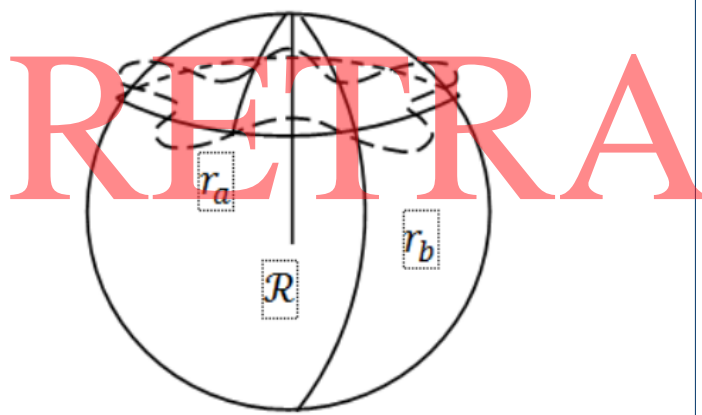

Figure 2: Lorentz contraction of the Bohr circumference which restricts the top part area and Lorentz contraction of the circumference which become zero at the south pole. De Broglie wave appears (e.g. for $n=5$ ).

on the $\frac{v_{a}}{r_{a}}=\frac{v_{b}}{r_{b}}$, we get the relation $\frac{r_{a}}{r_{b}}=\frac{v_{a}}{v_{b}}=\frac{v_{n}}{c}=\frac{a}{n}$. The area of the small disk is $S \odot_{a}=\sqrt{1-\frac{\alpha^{2}}{n^{2}}} \pi r_{a}^{2}$ and for the big disk is $S \odot_{b}=\sqrt{1-\frac{\alpha^{2}}{n^{2}}} \pi r_{b}^{2}$ with $S \odot_{b}=S_{B}$ and $S_{B}=4 \pi \Re^{2}$.

Further, through $\frac{r_{a}}{r_{b}}=\frac{v_{a}}{v_{b}}=\frac{v_{n}}{c}=\frac{a}{n}$, we get the relation between the big disk area and the small disk area as $S \odot_{b}=\sqrt{1-\frac{\alpha^{2}}{n^{2}}} \pi \frac{n^{2}}{\alpha^{2}} r_{a}^{2}$, and so $S \odot_{b}=\frac{n^{2}}{\alpha^{2}} S \odot_{a}$. Because the big disk area is equal to the sphere area with radius $\Re$, we obtain the relation $S \odot_{a}=\frac{\alpha^{2}}{n^{2}} 4 \pi \Re^{2}$ such a way that can be formed as

$$
\frac{S \odot_{n}}{S_{B}}=\frac{\alpha^{2}}{n^{2}}
$$

This relation prevails for all $n$ value which shows the ratio of Bohr atomic area on the sphere with respect to the sphere itself. This ratio connects with the fine structure depending on the $n$.

For determine how great the magnitude of space curvature where the Bohr atomic model is over there, previously we must look for the relation between Bohr radius $r_{a}$ and sphere radius $\Re$. From $S \odot_{a}=$ $\sqrt{1-\frac{\alpha^{2}}{n^{2}}} \pi r_{a}^{2}$ and $S \odot_{a}=\frac{\alpha^{2}}{n^{2}} 4 \pi \Re^{2}$ we get the relation

$$
\Re_{n}^{2}=\frac{n^{2}}{\alpha^{2}} \frac{\sqrt{1-\frac{\alpha^{2}}{n^{2}}}}{4} r_{n}^{2}
$$

Then by using Bohr radius value i.e. $0,53 \times 10^{-10} \mathrm{~m}$ for $n=1$, we get $\Re$ value is about $36,31 \times 10^{-10} \mathrm{~m}$. The sphere radiuses value where is the Bohr atomic model on it is $68,5 \mathrm{Bohr}$ radius itself. Then for determine the curvature, we use Ricci Scalar of the sphere [7], i.e.

So, the curvature value is $0,00152 \times 10^{20} \mathrm{~m}^{-2}$. This value may-be-able to be a standard to study curvature problem in the atomic level. Is this curvature value strong enough to give gravitational effect in the atomic scale?. Yes, the curvature's value as great as this can be felt by the Bohr atomic model itself remind that $\alpha$ can give effect to the forming of the fine structure in the flat coordinate.

Here also we ask that if the space curvature is properly as the consequence of the Bohr atomic model with relativistic correction, so why the atom chooses the flat space rather than the curve one?. The fine structure in reality describes spin-orbit coupling phenomenon which occurs only in the flat space as it is predicted by Dirac equation in atomic model. From the vector analysis, spin-orbit coupling can only occur if the space is Euclidean. This coupling is meaningless in the curve space. Further, if we view electron as the particle without spin, the phenomenon which occurs as the relativistic effect is the eliptic orbit with Thomas precission. It is the Bohr-Sommerfeld model. Two atomic models above are not in the curve space. The curvature effect of the Bohr atom may be occur in nature if there is a situation which can break the spin-orbit coupling phenomenon and 
Thomas precission. It is equivalent to the breaking the notion of the flat space. However, the curvature of the Bohr atomic model in theoretical point of view is the usefull concept to understand how much the curvature value in the coupling constant representation. This theory tells that how strong the fine structure is able to give curvature effect in the coordinate.

Further, based on the Bohr postulate, atom changes from one state to another by absorbing or emitting a quantum of light. In relativistic version, the difference of energy state have to posses additional term that contains the coupling constant as the Bohr atomic model correction according to Eq. (1) and (2). So we write this condition as follow:

$$
\Delta E=E_{f}-E_{i}=\hbar \omega+\Delta \varepsilon(\alpha)
$$

In the curve space, the difference of energy will manifest the difference of the curvature. When atom change the state, atom will change from such a curvature to another by releasing the entity which has information of curvature difference. The change of the curvature disturb the space-time coordinate and manifest quantum of gravitation. So we can view the quantum aspect of gravitation, i.e. the quantum aspect of the curvature that can move in the space-time coordinate. It suppose able to be connected with the concept of graviton. The curvature's change takes the form as follow:

$$
R_{f}-R_{i}=\frac{8 \alpha^{2}}{\alpha_{0}^{2}}\left(\frac{1}{n_{f}^{6} \sqrt{1-\frac{\alpha^{2}}{n_{f}^{2}}}}-\frac{1}{n_{i}^{6} \sqrt{1-\frac{\alpha^{2}}{n_{i}^{2}}}}\right)(10)
$$

Here, $\frac{8 \alpha^{2}}{a_{0}^{2}}$ is the constant of the curvature which is proposed. This constant is the standard value to measure how large the curvature in the atom and to state all the curvature's value in the gravitational study.

I see that this change can be connected with the concept of quantum energy based on the Bohr postulate in Eq. (9). The quantum of curvature in the quantum energy can be stated as

$$
\Delta \varepsilon(\alpha)=\varepsilon_{f}-\varepsilon_{i}=\hbar \kappa\left(R_{f}-R_{i}\right)
$$

This value is proportional to the quantum energy correction of light from Eq. (9). The $\kappa$ is equivalence quantity whose value is negative, but it does not mean that the curvature is also negative. The negative sign comes from the concept of negative energy because of the bounded state. It means that when the photon is absorbed in the Bohr atomic model, the space curvature change from the higher value to the lower one. So, a quantum of space curvature is also absorbed. Then, when a photon is emitted, a quantum of space is also emitted. It is like the concept of emition-absorption in the Bohr atomic model. The value of $R_{f}-R_{i}=\Delta R$ is negative when the transition from the higher curvature to the lower one and vice versa. On another hand, the $\kappa$ is the quantity which tells how the energy $\Delta \varepsilon \alpha$ can warp the surface such a way that it has the large curvature $\Delta R$. It is like the mechanism of Einstein field equation, but the clear form of $\kappa$ is not yet known. If the value of $\kappa$ is high, so the $\Delta R$ value is low and so for $R_{n}$. It can be considered as a reason of why the atom choose the flat space instead the curve space. The $\kappa$ has an equivalent characteristic to the flat space which manifests spin-orbit coupling and Thomas precission. Probably, there is such a mechanism which makes both mentioned phenomena decrease such a way that can be indicated in decreasing of $\kappa$. As the consequence, $R_{n}$ increases.

Further, we can express Eq. (9) in the space curvature as follow:

$$
\Delta E=\hbar \omega+\Delta \varepsilon(\alpha)=\hbar \omega+\hbar \kappa\left(R_{f}-R_{i}\right)
$$

It means that photon energy of relativistic Bohr atom between two states minus it's non-relativistic gives the value of energy which relates to the space curvature. We may able to approach the concept of "graviton" from here. But we have to be carefully, remind that the photon is the energy transfer in the space-time coordinate, while the graviton is the entity which has to be connected to the curvature of space-time itself. Graviton also moves with the speed of light. According to my study, "graviton" is the entity of quantum curvature which propagates in the space-time coordinate. Every quantum of gravitation brings quantum of curvature. As we have known that photon manifests the frequency, "graviton" manifests the space-time curvature. From theoretical point of view, we can describe this phenomenon as follow: 


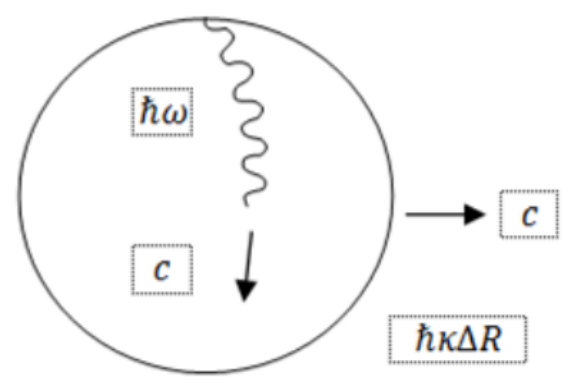

Figure 3: Quantum of the curvature and photon motion during emission or absorption of Bohr atomic model with Ehrenfest paradox.

Photon with energy $\hbar \omega$ is on the sphere which correspond to the energy $\hbar \kappa \Delta R$. This phenomenon occurs because there is no any flat space for the photon in this situation. Photon moves with velocity $c$ on the moving sphere. This sphere also moves with speed $c$. In this theory, the direction of the photon propagation on the sphere is arbitrary and does not affect the moving curvature. Principally, both photon and space curvature move together. The important concept is_a moving quantum curvature with energy $\hbar \kappa \Delta R$. We can view this as the standard unit to state gravitational wave.

Gravitational wave which is predicted by general theory of relativity is in the classical sense [8]. We can assume that the gravitational wave is built by the number of quantum curvature as we have understand for other field waves according to the quantum field theory. For example: electromagnetic wave. This wave contains photons. Intensity of classical electromagnetic wave is depend on amount of photons $[9,10]$ and so does gravitational wave. But we have to be carefully, because in the case of electromagnetic wave, frequency does not change when intensity increases, while the situation is different for the gravitational wave. The unification of amount of the higher quantum curvatures in Figure 3 builds the gravitational wave in the classical form with the lower curvature. So, when intensity of the gravitational wave increases, the curvature effect to be weak.

Further, we can view every Bohr curvature energy $\varepsilon \alpha$ in the context of mass-energy equivalence, so we write that

$$
m c^{2}=\hbar \kappa R_{n}
$$

This equation express the energy of Bohr atomic curvature with $m$ is a theoretic mass that causes the curvature effect. This equation also tells that there is a mechanism from dynamical aspect for a little energy and/or mass in the atomic scale to make the large curvature from the macroscopic point of view. In our scale we view that the large curvature is contained in the small dimension, i.e. the dimension of atom. The value of $R_{n}$ is not too strong for the atomic dimension but for macroscopic dimension, this value is too strong. How is the significant of the curvature is depend on the scale of the object. Then, we express the mass density as $\rho c^{2}=\hbar \kappa^{\prime} R_{n}$, in such a way that we can relate this concept to the Einstein field equation which tells the present of the density to form the space-time curvature. The $\kappa^{\prime}$ is the $\kappa$ per unit volume which the value can be determined from Eq.(14), while the density of this equation is determined from the second term of Eq.(9). Notice! that in Eq.(14), the energy density is in the three dimension, while the surfase which is curved is in the two dimension. In the context of Einsten field equation, the energy-momentum tensor rank has to be equal to the Ricci tensor rank. This equation is also four space-time dimension. So, the situation is described by Eq.(14) is not exactly equal to that by Einstein field equation, but we can modify $R_{n}$ as the Ricci scalar of the sphere to the four dimension of space-time. Hence, we also have to modify $\kappa^{\prime}$ to another quantity, e.g. $\sigma$ and replace the density to energy-momentum scalar as follow

$$
T_{n}=\hbar \sigma R_{n}^{(4)}
$$

On another hand, when we do the tensor contraction to the Einstein field equation, we get

$$
R=2 \frac{8 \pi G}{c^{3}} T
$$

So, there is a relation between Eq.(14) and (15). Equation (14) tells that the density can form the curvature like Eq.(15), except that Eq.(14) is expressed in the Planck constant. We can use Eq.(14) in the context of miniblackhole. Miniblackhole has the tiny mass but the large density such a way that causing the large curvature [7]. Equation (14) can be stated in the curvature of Eq. (13) previously which contains the fine structure.

Principally, gravitation is weak for the large scale but to be strong for the atomic scale. It is properly that the gravitational wave which is produced in calestial object is not significant to the atomic scale. In other words, if we will reach the quantum aspect of the gravitation, so we have to be able to produce the very strong gravitational field from macroscopic point of view, such a way that it gives the significant curvature effect 
to the atomic scale. This situation possibly occurs in the early universe expansion, when gravitational field is strong for the atoms.

Another interesting consequence of Ehrenfest paradox in the Bohr atomic model is that we can take the tiny tangen space from the Figure 3 to explain the Equivalence Principle. The photon moves in curved space as we have ever seen in ordinary Equivalence Principle. Figure 3 shows the foundamental relationship between photon and the curvature, i.e between photon and gravitation. It gives the viewpoint about Equivalence Principle for the case of photon-gravitation in the smallest unit. In this situation, Equivalence Principle is in the quantization phenomenon. Hence, it is equivalence to the Quantization of the Equivalence Principle itself. The investigation of this subject can be done beyond this study.

\section{Conclusion}

All the procedures that have been done to quantize gravitation are based on the Bohr atomic model scenario. This study is the theoretical point of view in which can be considered to quantize gravitation. The concept of graviton theoretically can be understood if we view the Bohr atomic model in the curved space as it's relativistic effect. Coordinate curvature can be stated by the fine structure. The curvature in the Bohr atomic model can be a standard to measure how strong the curvature of all system are?, by comparing them with the value of Bohr's atomic curvature. The change of the curvature will disturb the space-time coordinate in the the form of curvature propagation in quantum energy, i.e. $\Delta \varepsilon=\hbar \kappa \Delta R$. It can be the alternative manner to enlarge the theory about unification between quantum and gravitation.

\section{Author}

1 Fima Ardianto Putra

Dari :

(1) Department of Physics, Universitas Jenderal Soedirman

Pustaka

1. Eisberg R, Resnic R. Quantum Physic of Atom, Molecul, Solid, Nuclei, and Particle. John Wiley \& Sons, Inc.; 1985.

2. Winterberg F, Naturforsch Z. 53a; 1998.

3. Beiser A. Concept of Modern Physics. 6th ed. McGraw-Hill Companies, Inc.; 1992.

4. Ryder L. Introduction to General Relativity. United Kingdom: Cambridge University Press; 2009.

5. D H, Resnic R. Fisika. Jakarta: Erlangga; 1985.

6. Giancolli C, Douglas. Fisika. Jakarta: Erlangga; 2001.

7. Hobson MP, Efstathlou G, Lasenby N. General Relativity: An Introduction for Physicists. Cambridge: Cambridge; 2008

8. Ortin T. Gravity and String. Cambridge: Cambridge; 2004.
9. Ryder LH. Quantum Field Theory. Cambridge: Cambridge University Press; 1985

10. Mandl, F, Shaw GP. Quantum Field Theory. 2nd ed. John Wiley \& Sons, Inc.; 2010.

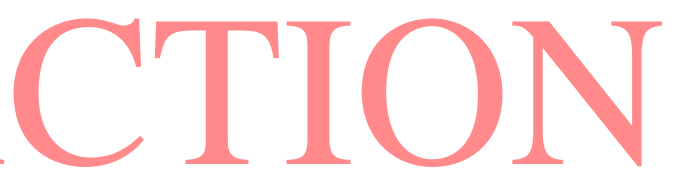

